\title{
Testosteronkiller
}

\section{Gefährdet Schlafmangel die Männlichkeit?}

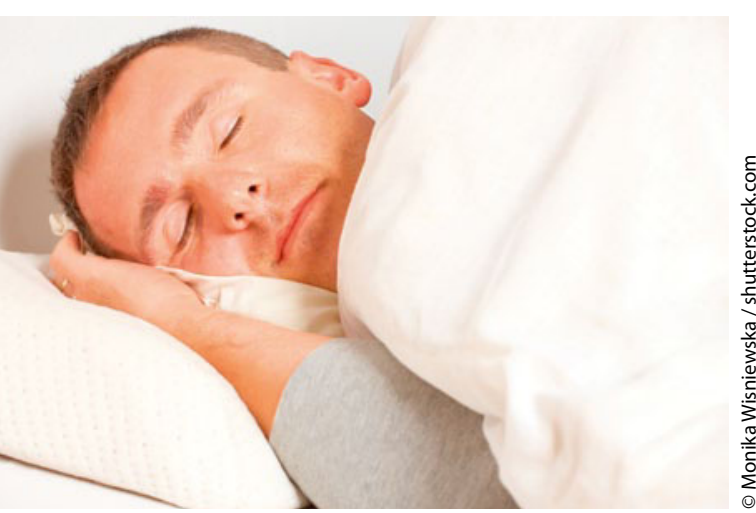

Anti-Aging für die Hormone ausreichend Schlaf

— In einer Studie sank der Testosteronwert von jungen gesunden Männern um 10-15\%, wenn sie eine Woche lang mit nur fünf Stunden Nachtschlaf auskommen mussten. Zunächst durften die zehn Probanden eine Woche lang acht Stunden pro Nacht zuhause schlafen. Die nachfolgenden elf Tage verbrachten sie im Labor, wo man ihnen die ersten drei Nächte rund neun Stunden Nachtschlaf gönnte. Danach wurde die Nachtruhe unter Überwachung der verschiedenen Schlafphasen auf knapp fünf Stunden gekappt. Nach der zweiten langen und der siebten kurzen Nacht wurden jeweils für 24
Stunden alle 20 bis 30 Minuten Blutproben zur Bestimmung von Testosteron und Kortisol entnommen.

Bei der Auswertung der Proben stellte sich heraus, dass nach den Nächten mit weniger Schlaf die Testosteronwerte in den Wachzeiten zwischen 8 und 22 Uhr im Schnitt deutlich niedriger lagen als an den Tagen nach ausreichend Bettruhe (16,5 vs. $18,4 \mathrm{nmol} / \mathrm{l})$. Am deutlichsten zeigte sich dieser Unterschied zwischen 14 und $22 \mathrm{Uhr}$ ( $15,5 \mathrm{vs.} 17,9 \mathrm{nmol} / \mathrm{l})$. Je länger der Schlafentzug insgesamt dauerte, desto mehr verspürten die Männer Auswirkungen auf ihre allgemeine Vitalität und ihr Wohlbefinden.

Bei den Kortisol-Tagesprofilen dagegen fanden sich keine Unterschiede zwischen Viel- und Wenigschlaftagen. So scheint der Testosteronabfall keine Stressreaktion zu sein, die die Gonadenfunktion beeinflusst haben könnte.

Bedenkt man, dass der Mann allein durch seinen normalen Alterungsprozess jährlich etwa $1-2 \%$ seines Testosterons einbüßt, könnte ein ausreichender Nachtschlaf durchaus auch der Männlichkeit dienen. Allerdings seien größere Studien erforderlich, um die genauen Zusammenhänge zwischen Schlaf und Testosteronspiegel zu untersuchen, betonen die Autoren.

\section{Schlaf und Beischlaf}

\section{Eine Impotenz kommt selten allein}

— Mangelhafte Performance im Bett scheint ein komplexes Problem zu sein: Die Wahrscheinlichkeit, an obstruktiver Schlafapnoe zu leiden, liegt für Männer mit erektiler Dysfunktion (ED) doppelt so hoch wie für Normalpotente. Dies haben US-amerikanische Mediziner in der bisher größten Studie ihrer Art herausgefunden.

An der Untersuchung waren 870 Männer mit einem Durchschnittsalter von 47 Jahren beteiligt. Der Body-Mass-Index der Probanden erreichte den stattlichen Mittelwert von 30,2. 63\% der Männer litten an Schlafapnoe, 5,6\% waren Diabetiker und 29\% rauchten. Nachdem die Forscher Alter und Zusatzerkrankungen berücksichtigt und die Teilnehmer vergleichbaren Gruppen zugeordnet hatten, suchten sie nach der Verbindung von erektiler Dysfunktion und Apnoe. Eine ED verdoppelte dabei die Wahrscheinlichkeit, dass Betroffene auch eine Apnoediagnose erhalten hatten. Und je schwerer die ED ausgeprägt war, desto höher fiel das Apnoerisiko aus.

Männer mit ED sollten daher unbedingt auch auf obstruktive Schlafapnoe untersucht werden, lautete das Fazit der Wissenschaftler. 